\title{
Dynamic Access Network Selection with QoS Parameters Estimation: A Step Closer to ABC
}

\author{
Eng Hwee Ong, Jamil Y. Khan \\ School of Electrical Engineering and Computer Science \\ University of Newcastle, Australia, NSW 2308 \\ Email: enghwee.ong@studentmail.newcastle.edu.au,jamil.khan@ newcastle.edu.au
}

\begin{abstract}
Always best connected (ABC) services allows multimode mobile terminals to stay connected to the best available networks, at anytime according to user preferences. One of the key aspects in realizing such $\mathrm{ABC}$ service is mainly attributed to an effective and dynamic access network selection process. However, most of the previous works consider the access network selection process as a static optimization problem which fails to address the dynamic QoS conditions intrinsic in wireless networks. One of the main challenges remains as no efficient way in obtaining dynamic QoS parameters such as packet delay, packet loss and jitter. In this paper, we proposed a novel dynamic access network selection algorithm capable of adapting to prevailing network conditions. Our algorithm is a dual stage estimation process where network selection performed using sequential Bayesian estimation relies on dynamic QoS parameters estimated through bootstrap approximation. Simulations demonstrate the effectiveness of our proposed algorithm which outperforms static optimization approach in a highly efficient manner.
\end{abstract}

\section{INTRODUCTION}

The seminal work of Gustafsson et al. [1] on ABC networks and services has gained much attention recently and has been adopted as one of the main drivers of $4 \mathrm{G}$ evolution. Together with the demands of ubiquitous connectivity and high data rates in $4 \mathrm{G}$ networks, the importance of interworking between existing and possibly new radio access technologies (RAT) are prevalent since the available resources of any single network is insufficient to meet such requirements. In a nutshell, $4 \mathrm{G}$ has been envisaged as a congregation of complementary heterogeneous RATs where seamless services could be provisioned dynamically through the most efficient network based on user preferences and prevailing network conditions.

Further, 4G would converge into an all-IP network for integrating different RATs [2]. With the introduction of new RAT such as worldwide interoperability for microwave access (WiMAX) in the near future, we consider a heterogeneous IP-based 4G architecture comprising of UMTS, WLAN and WiMAX as illustrated in Fig. 1. The IP-based core network enable easy exploitation of existing mobile IP techniques to achieve seamless handover with QoS support [3]. However, the access network heterogeneity demands an efficient network selection scheme such that users can be best connected through multi-mode terminals. We postulate that users should be best connected not only during initial network access but also throughout the entire duration of their connection. This could be addressed by performing QoS-based handover to next best network that will satisfy the user service QoS profile. However, this requires adaptation to prevailing network conditions.

Most of the previous research works relating to $\mathrm{ABC}$ service either consider access network selection as static optimization problem which did not sufficiently address the dynamic nature of the network conditions or circumvent obtaining dynamic QoS information altogether. Chen et al. [3] introduces a new 4G architecture to overcome the challenges of collecting real-time QoS information necessary for network selection. Instead, their QoS information are gathered through a threetier hierarchical fashion susceptible to single point of failure and increased latency. Nguyen-Vuong et al. [4] depends on SNR/SNIR to capture the varying network conditions. However this is only effective in reflecting the wireless channel quality rather than network conditions which may be a consequence of both channel quality and network congestions.

Song et al. [5] proposed an access network selection strategy using analytic hierarchy process (AHP) and grey relational analysis (GRA) based on extensive QoS information. However, the QoS information gathered using cross-layer signaling manifest in complexity of its protocol stack and non-deterministic processing latency. Moreover, the access network selection is triggered only when the service class or access point changes which undermined the varying network conditions otherwise. Zhang et al. [6] introduces MAC layer sensing based on fixed relationship of network allocation vector (NAV) and bandwidth/access delay to estimate network conditions for a given packet length. However, this technique limited by the use of NAV and cannot be extended to non-802.11 networks.

The key limitation to ongoing research remains as no efficient way to obtain dynamic QoS information required for network selection. We argue that the main challenges are low latency of data processing for real-time applications, reliability of data and size of data to be considered pragmatic. Since $4 \mathrm{G}$ networks would be predominantly based on multimedia traffic, it is also important to consider the effects of self-similar traffic apparent in the IP-based core. These motivate us to estimate the probability distribution of dynamic QoS parameters by statistical inference since it is often unrealistic to observe the entire population. The remainder of the paper is organized as follows. Section II describes our proposed dynamic access network selection algorithm. Section III illustrates the simulation model and simulation scenarios. Section IV presents the simulation results and Section V concludes the paper. 


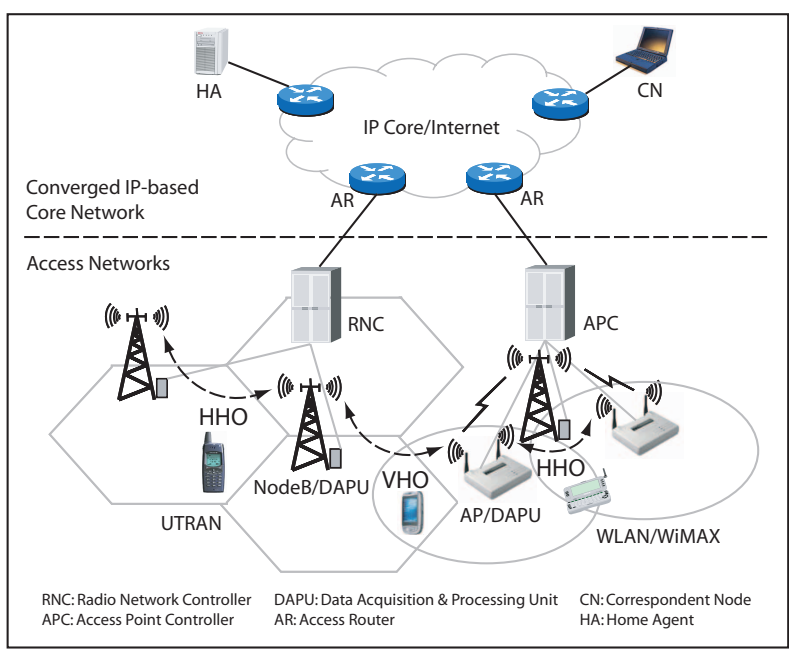

Fig. 1. 4G architecture.

\section{Proposed Dynamic Access Network Selection ALGORITHM}

The concept of our dynamic access network selection algorithm is a dual stage estimation process that supports terminaloriented, network-assisted (TONA) handover architecture as depicted in Fig. 2. QoS parameters are in general nonstationary, however they can be considered as stationary when observed over a short time. Our motivation is to account for the effects of non-stationary components using sequential Bayesian estimation which relies on bootstrap approximation to estimate the short-term stationary data. The shaded blocks refer to network entities while the unshaded blocks refer to terminal entities. The blocks in dash lines complete the support for $\mathrm{ABC}$ services by considering both network conditions and user preference during network selection while maintaining optimal traffic distribution through admission control. However, these are outside the scope of this work.

The TONA handover architecture can be triggered by two events, viz. initial access to networks where user would choose the optimal network according to their service QoS profile and handover when the service QoS of user drop below a predefined threshold. In the first stage, we perform bootstrap approximation to estimate the dynamic QoS parameters in data acquisition and processing unit (DAPU) as part of the base station (BS) and/or access point (AP). For the second stage, we perform sequential Bayesian estimation in the mobile terminal (MT) by leveraging on the broadcasted QoS parameter estimates. We note that our solution is not restricted to the control of heterogeneous systems during vertical handover (VHO) but also performance enhancement of homogeneous systems during horizontal handover (HHO).

We believe that $4 \mathrm{G}$ networks would be predominately based on multimedia traffic, and henceforth identified the critical QoS parameters as packet delay, packet loss and jitter. The estimation of the average packet delay from a given network is exemplified in this section. Similar approaches could be subsequently used to estimate both the average packet loss

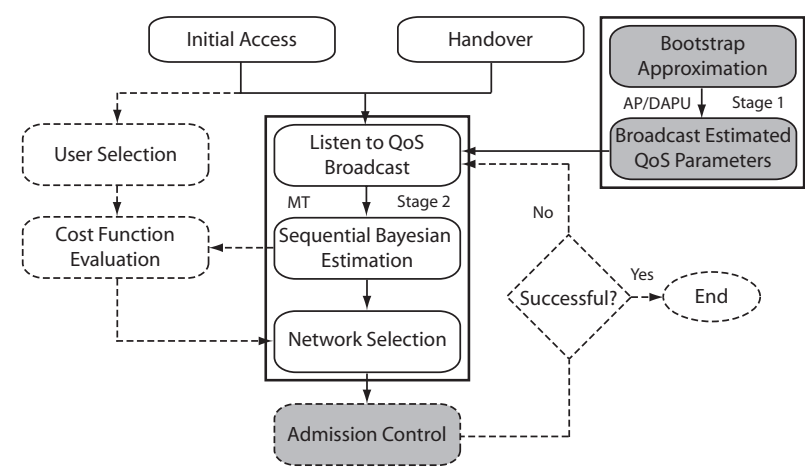

Fig. 2. Terminal-oriented, network-assisted (TONA) handover architecture.

and jitter for any given networks. Normal Approximation has been used for network delay estimation by Gibbon [7] to implement a scheduler which manages the retrieval of distributed multimedia data. However, there exist two potential problems. First, Central Limit Theorem (CLT) does not tell us how large should be our sample size before it converges to normal distribution. Particularly when dealing with realtime applications, the assumption of large samples cannot be established because of time constraints and limiting data. Second, we have adopted sample median as an estimator of average values for any density functions due to its robustness when considering self-similar traffic which manifest as heavytailed distributions. However, CLT which holds for sample mean does not apply to sample median. Therefore, there is no equivalent formula $\sigma(F)=\left[\mu_{2}(F) / n\right]^{1 / 2}$ that expressed standard error of sample mean as a simple function of the sampling distribution for the case of sample median.

For these reasons, we have adopted the bootstrap method developed by Efron [8] for estimating the probability distributions of critical QoS parameters from the acquired data itself without the need for unrealistic or unverifiable assumptions. Moreover, bootstrap method have been shown to successfully estimate the variance of a sample median which has known to fail with the jackknife approach. Notably, bootstrap method has been widely used in multitude of disciplines such as signal processing, biomedical engineering, environmental and geophysical research to approximate the probability distribution of an estimator or its higher order statistics of some form. A comprehensive treatment of bootstrap applications in signal processing could be found in [9].

\section{A. QoS Parameter Estimation with Bootstrap Approximation}

The bootstrap method is a computer-based, non-parametric approach where no assumptions are made on the underlying population from which the samples are collected. Here, we approximate the measured packet delay as independent and identically distributed (i.i.d) during our data acquisition window. Although, network packets traveling between a certain source and destination within the same network cannot be truly independent and statistical distribution of the network QoS parameters which we seek to sample data from would not 
be truly identical due to varying channel conditions. However, it is an assumption that approximates the actual conditions.

Suppose we want to conduct inference about the unknown parameter $\theta$ from a true population $F$ which we denote $\theta=\theta(F)$. We can consider the bootstrap method for the onesample situation where random samples $x_{i}$ are drawn from a single unknown distribution function $F$ forming the original data set $X=\left(x_{1}, x_{2}, \ldots, x_{n}\right)$. We used $F \rightarrow\left(x_{1}, x_{2}, \ldots, x_{n}\right)$ to represent that $X=\left(x_{1}, x_{2}, \ldots, x_{n}\right)$ is a random sample of size $n$ from a population with an unknown probability distribution $F$. An original data set provides a simple estimate of the entire population based on the assumption that it constitutes the underlying distribution. The discrete empirical distribution $\hat{F}$ is then formed by assigning a probability mass of $1 / n$ on each $x_{i}$ of the original data set, such that each $x_{i}$ has an equal likelihood of being chosen when resampling from $\hat{F}$. The bootstrap data set $X^{*}=\left(x_{1}^{*}, x_{2}^{*}, \ldots, x_{n}^{*}\right)$ is subsequently defined to be a random sample of size $n$ drawn with replacement from $\hat{F}$. Similarly, we used $\hat{F} \rightarrow\left(x_{1}^{*}, x_{2}^{*}, \ldots, x_{n}^{*}\right)$ to represent that $X^{*}=\left(x_{1}^{*}, x_{2}^{*}, \ldots, x_{n}^{*}\right)$ is a resampled random sample of size $n$ from empirical distribution $\hat{F}$.

The bootstrap estimate is denoted by $\hat{\theta}=\theta(\hat{F})$ where, $\theta(\hat{F})$ is the plug-in estimate that uses the empirical distribution $F$ in place of unknown distribution $F$. Since we are interested in estimating some parameter $\theta$ by calculating a statistic from a random sample, correspondingly we can calculate the same statistic from a bootstrap data set $X^{*}$ to obtain the bootstrap replication of $\hat{\theta}$,

$$
\hat{\theta}_{\mathrm{b}}^{*}=s\left(X_{b}^{*}\right), \quad b=1,2,3, \cdots, B,
$$

where $X_{b}^{*}=\mathrm{b}$-th bootstrap data set of B independent bootstrap data sets. For example, our statistic of interest $s(X)$ is the sample median $\tilde{x}$ hence, $s\left(X^{*}\right)$ is the median of the bootstrap data set $\tilde{x}^{*}=x_{i+1}^{*}$ from the ordered sample values $x_{1}^{*}<$ $x_{2}^{*}<\ldots<x_{2 i+1}^{*}$. Hence, $\mathrm{B}$ bootstrap replicates provide us an estimate of the $\hat{\theta}$ distribution and its standard deviation is used to estimate the standard error for $\hat{\theta}$ given by,

$$
S \hat{E}_{B}(\hat{\theta})=\sqrt{\frac{1}{B-1} \sum_{b=1}^{B}\left(\hat{\theta}_{b}^{*}-\overline{\hat{\theta}}^{*}\right)^{2}}, \quad \overline{\hat{\theta}}^{*}=\frac{1}{B} \sum_{b=1}^{B} \hat{\theta}_{b}^{*} .
$$

Accordingly, we can now estimate the average packet delay distribution from B bootstrap replicates and the standard error of the average packet delay using Eq. 2, forming the parameter estimates of the average packet delay. The number of bootstrap replicates $50 \leq B \leq 200$ have been shown in [8] to be sufficient when estimating the standard error of a statistic. When sampling with replacement, there is a possibility that for some $x_{i}$ it would occur more than once or not at all. Earlier, we have assumed $\hat{F}$ as a suitable estimate for $F$. However, the discrete nature of $\hat{F}$ and resampling would manifest in jagged bootstrap replicates distribution of sample median. We mitigate this with a technique known as smooth bootstrap where bootstrap data sets are constructed from a smooth empirical distribution instead. Without loss of generality, we used $\hat{F}_{s} \rightarrow\left(y_{1}^{*}, y_{2}^{*}, \ldots, y_{n}^{*}\right)$ to represent that $Y^{*}=\left(y_{1}^{*}, y_{2}^{*}, \ldots, y_{n}^{*}\right)$ is a resampled random sample of size $n$ from smoothed empirical distribution $\hat{F}_{s}$.

\section{B. Network Selection with Sequential Bayesian Estimation}

Bayes decision is a fundamental statistical approach to many difficult data-modeling problems, providing a formal and intuitive way to make decision in presence of uncertainties. Suppose we wish to make an inference of the average packet delay described by unknown parameters $\left(\mu_{k}^{i}, \sigma_{k}^{2 i}\right)$ of network $i$ with respect to packet delay threshold over time $k$. Let $y_{i}=\left[y_{k}^{i}, y_{k+1}^{i}, \ldots, y_{N}^{i}\right]^{T}$, the measurement data at network $i$ denote the observed values of estimated average packet delay at times $k, k+1, \ldots, N$. We can then apply Bayes rule sequentially as,

$$
p\left(\mu_{k}^{i}, \sigma_{k}^{2 i} \mid y_{k}^{i}\right) \propto p\left(y_{k}^{i} \mid \mu_{k}^{i}, \sigma_{k}^{2 i}, y_{k-1}^{i}\right) p\left(\mu_{k}^{i}, \sigma_{k}^{2 i} \mid y_{k-1}^{i}\right) .
$$

Recall that packet delay measurements are acquired in nonoverlapping successive windows during our bootstrap approximation. Hence, we assume successive measurement windows are independent and the likelihood function simplifies to,

$$
p\left(y_{k}^{i} \mid \mu_{k}^{i}, \sigma_{k}^{2 i}, y_{k-1}^{i}\right)=p\left(y_{k}^{i} \mid \mu_{k}^{i}, \sigma_{k}^{2 i}\right) .
$$

We can invoke the use of conjugate prior distribution as the estimation is performed recursively and the likelihood function data estimated by our DAPU are normally distributed. The sampling variance of observation $y_{k}^{i}$ corresponds to standard error of our bootstrap estimates and is assumed to be constant. The conditional posterior distribution of $\mu_{k}^{i}$, given $\sigma^{2 i}$ can then be shown as [10],

$$
p\left(\mu_{k}^{i} \mid \sigma^{2 i}, y_{k}^{i}\right) \sim N\left(\hat{\mu}_{k}^{i}, \hat{\sigma}_{k}^{2 i}\right),
$$

where

$$
\hat{\mu}_{k}^{i}=\frac{\mu_{k-1}^{i} / \sigma_{k-1}^{2 i}+y_{k}^{i} / \sigma^{2 i}}{1 / \sigma_{k-1}^{2 i}+1 / \sigma^{2 i}}, \quad \hat{\sigma}_{k}^{2 i}=\frac{1}{1 / \sigma_{k-1}^{2 i}+1 / \sigma^{2 i}} .
$$

This numeric simplification is a merit of bootstrap approximation which results in normally distributed data. By adding information for the measurement data one point at a time, we essentially updates the posterior distribution as prior distribution for the next cycle of the sequential procedure. In another words, new QoS parameter estimates from our DAPU can be used to change our beliefs to reflect our better knowledge of the prevailing network conditions. However, it is often desirable to reset the estimation process when network condition stabilizes after each transition in such sequential estimation framework. The challenge in tracking non-stationary network conditions is to devise a mechanism which could reset the estimator optimally since old information about the network after it reaches its steady state is no longer useful. Therefore, it should be forgotten to allow convergence to new estimates. We have implemented a change detection scheme using cumulative sum (CUSUM) test [11], known in the context of quality control to detect any change of states and thereby resetting our Bayes estimator adaptively. 
The input of CUSUM test is the magnitude of the residuals normalized with respective to its standard deviation as,

$$
s_{k}^{i}=\frac{y_{k}^{i}-\hat{\mu}_{k-1}^{i}}{\sqrt{E\left[\left(y_{k}^{i}-\hat{\mu}_{k-1}^{i}\right)^{2}\right]}} .
$$

This normalization enables the same set of design parameters $(\delta, h)$ to be used for different scenarios. For two-sided CUSUM test, a pair of auxiliary test statistics $g_{k}^{i+}$ and $g_{k}^{i-}$ is necessary for detection of state changes in non-stationary network conditions as,

$$
\begin{aligned}
& g_{k}^{i+}=\max \left(g_{k-1}^{i+}+s_{k}^{i}-\delta, 0\right), \\
& g_{k}^{i-}=\max \left(g_{k-1}^{i-}-s_{k}^{i}-\delta, 0\right) .
\end{aligned}
$$

Test statistics are initialized with a starting value of zero and will start accumulating its residual as soon as the Bayes estimate deviates from the measurement data by more than the drift parameter $\delta$. A positive drift would result in accumulation of residual in $g_{k}^{i+}$ and a negative drift would result in accumulation of residual in $g_{k}^{i-}$. An alarm will then be triggered when either test statistics exceeds the alarm threshold $h$. After an alarm, the respective test statistic is cleared to zero and the Bayes estimator resets.

To quantify our Bayes estimates in terms of network quality, we compute the probability of estimated average packet delay in network $i$ being less than or equal to the packet delay threshold $\tau$ using the cumulative distribution function (CDF),

$$
F_{\mu_{k}^{i} \mid \sigma^{2 i}, y_{k}^{i}}(\tau)=\Phi\left(\frac{\tau-\hat{\mu}_{k}^{i}}{\hat{\sigma}_{k}^{2 i}}\right),
$$

where

$$
\Phi(\tau)=\frac{1}{\sqrt{2 \pi}} \int_{-\infty}^{\tau} \exp \left(-\frac{u^{2}}{2}\right) d u .
$$

The network with the highest probability will then be selected.

\section{Simulation Model}

Without loss of generality, we demonstrate the effectiveness of our dynamic access network selection algorithm using homogeneous wireless LAN 802.11b distributed coordination function (DCF) system in infrastructure mode with two access points (AP) as shown in Fig. 3. Note that the results would be irrespective of implementation in homogeneous or heterogeneous systems. The DAPU reside in each AP of the WLAN network and would perform online packet delay measurements. Timestamps are encapsulated in packets transmission by mobile terminals (MT) to enable uplink measurements, however this is unnecessary for downlink packets since measurements are taken from AP itself. When the DAPU acquired sufficient sample size of 200 samples, it would perform offline bootstrap Monte Carlo processing to estimate the probability distribution of the average packet delay. This estimated QoS parameters would then be encapsulated in the broadcast channel and transmitted in the downlink, where MT could listen and performs network selection using sequential Bayesian estimation.

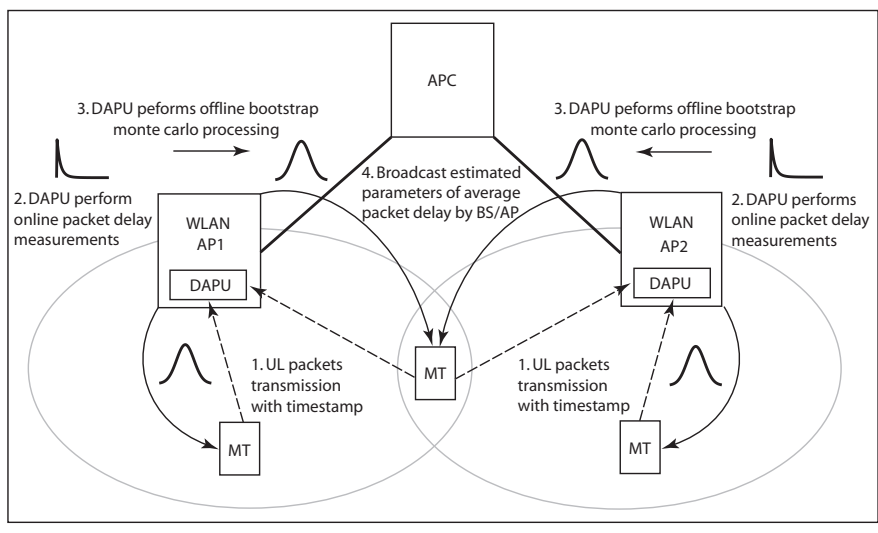

Fig. 3. Simulation model.

We assumed that MT has two multi-mode RF front-ends in order to listen to broadcast channel messages from both APs, which is aligned to the vision of future chip set solutions [12] for realizing $\mathrm{ABC}$ services. The offline bootstrap Monte Carlo procedures of the DAPU are as follow:

Step 1. Obtain the original data set $X=\left(x_{1}, x_{2}, \ldots, x_{n}\right)$ through data acquisition.

Step 2. Obtain bootstrap data set $Y_{b}^{*}=\left(y_{1_{b}}^{*}, y_{2_{b}}^{*}, \ldots, y_{n_{b}}^{*}\right)$, each of $n$ data values from smoothed empirical distribution $\hat{F}_{s}$ by sampling with replacement.

Step 3. Calculate the bootstrap replicates $\hat{\theta}_{b}^{*}$ by computing the sample median, for each corresponding bootstrap data set obtained in step 2.

Step 4. Repeat step 2 through 3 B times.

Step 5. Use the distribution of $B$ bootstrap replicates $\hat{\theta}^{*}$ as parameter estimates to the distribution of $\hat{\theta}$.

The simulation model was developed using OPNET $^{\mathrm{TM}}$ Modeler ${ }^{\circledR} 12.1$ with Wireless Module. We have performed minor modifications to the existing DCF model without departure from the IEEE $802.11 \mathrm{~b}$ specifications for integration with our custom DAPU model. We further assumed that all stations (STA) are within the transmission range of each other i.e no hidden terminals and excluded RTS/CTS mechanism from our simulation. We then evaluate the robustness of our algorithm by utilizing three standard application models, viz. VoIP, video conferencing and video streaming to generate real-time traffic.

In our first simulation scenario, we have 4 STAs per AP with mixed traffic load. One pair of STAs transmit VoIP traffic and two pairs of STAs transmit video conferencing traffic where each VoIP and video conferencing session contains both uplink and downlink streams. The fourth STA of each AP do not transmit any traffic. This is to investigate the performance of our algorithm under extreme condition where both networks have almost similar load and packet delay. In the second simulation scenario, the fourth STA of each AP engages in video streaming at two distinct time to increase the load of respective APs. In infrastructure mode, the downlink becomes the bottleneck [13] for two-way communications such as VoIP and video conferencing. Hence, the packet delay of interest is taken as media access (MAC) delay experience by the AP. 

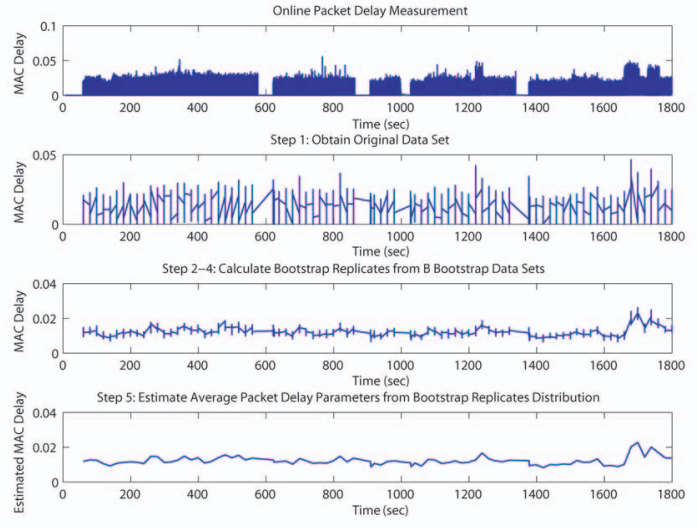

Fig. 4. Bootstrap Monte Carlo procedures in DAPU of AP2.

\section{Simulation Results}

The simulation results at different stages of our DAPU residing in AP2 is presented in Fig. 4. The DAPU first perform online packet delay measurement of AP2 MAC delay. The offline bootstrap Monte Carlo processing is triggered every 20 seconds which result in an original data set of 200 samples and consequently 200 bootstrap data sets after sampling with replacement from the original data set. Bootstrap replicate for each corresponding bootstrap data set is then calculated by computing the sample median. Finally, the average packet delay is estimated from the sampling distribution of the 200 bootstrap replicates which evidently shows that our DAPU is able to capture the dynamic behavior of the prevailing MAC delay. Next, we showed in Fig 5 that the sampling distribution of bootstrap replicates follows a normal distribution without recourse to CLT. This is a result of employing smooth bootstrap and it follows that sample median of any continuous function exhibit asymptotic normality [14]. This important property provides analytical tractability when incorporating the average packet delay estimates from the DAPU in our sequential Bayesian estimation.

The outcome of our dynamic network selection algorithm for simulation scenario 1 is depicted in Fig. 6. We refer to our result as Bayes selection scheme, which selects the best AP having MAC delay less than or equal to the packet delay threshold. A packet delay threshold of $12 \mathrm{~ms}$ which coincides with the mean level of average packet delay in both APs, referred as gray decision zone is chosen. We then contrast this against simple selection scheme, which selects any AP having the lowest MAC delay. The virtue of our approach can be seen from the effectiveness in preventing unnecessary network re-selection which could well result in 'ping pong' handover if triggered. We point out that the result of simple selection scheme is indicative of static cost function optimization schemes performance reported in [3-5], where network with criterion(s) that maximize or minimize the cost function will be selected. Our algorithm can make a more informed selection by considering both prior information

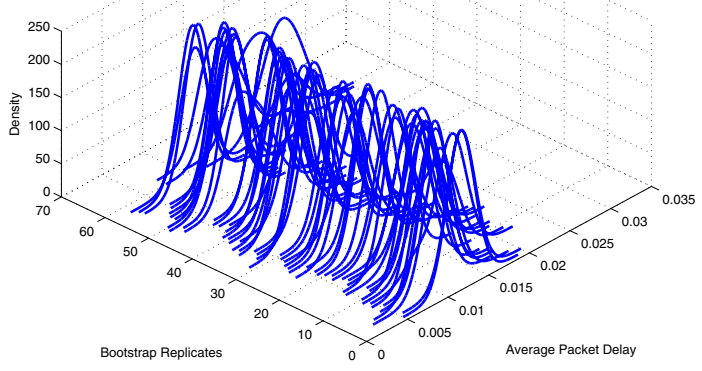

Fig. 5. Sampling distribution of bootstrap replicates at AP2.
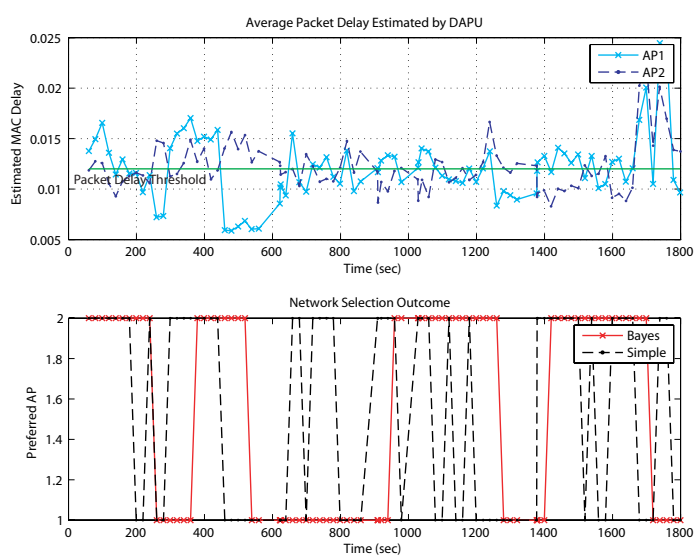

Fig. 6. Network selection outcome of simulation scenario 1 .

and measurement data during network selection whereas the former consider only current (static) outcome of their cost function evaluation. This is crucial for achieving stability of the system and minimizes unjustified usage of scarce resources if unnecessary handover can be precluded. For simulation scenario 2, we have raised the packet delay threshold to $40 \mathrm{~ms}$ which again corresponds to the gray decision zone in both APs, since the fourth STA of each AP now participate in video streaming respectively. It is apparent from Fig. 7 that our algorithm still yields better performance than simple selection in terms of more reliable network selection outcome.

We have shown the robustness of our dynamic network selection algorithm using two scenarios indicative of the worst case as in scenario 1 and the general case as in scenario 2 . We further conduct a comparative study of our algorithm against four other schemes. The simple scheme selects whichever AP having the lowest MAC delay. The static scheme selects the AP whose average packet delay maximizes the cumulative distribution function in Eq. 9. The two-state Markov chain scheme is implemented as in [15]. Lastly, the moving average scheme selects AP in similar fashion to static scheme with the exception that mean of average packet delay are first computed over a short window. In this study, we used the estimated MAC delay from our previous simulation scenarios 

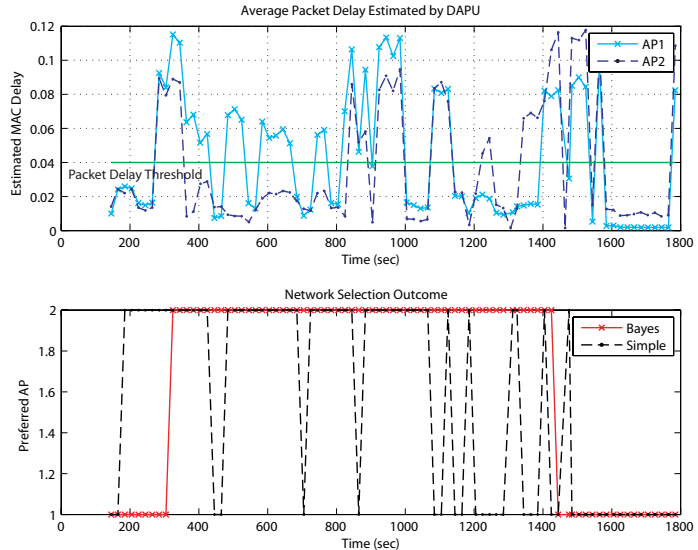

Fig. 7. Network selection outcome of simulation scenario 2 .

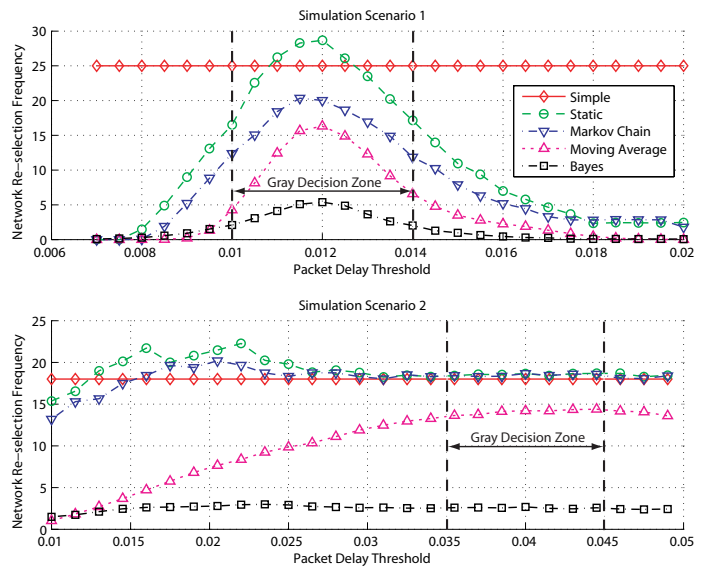

Fig. 8. Network selection algorithms performance.

to generate uniformly distributed pseudo-random scenarios of different MAC delay realization. We then compare network re-selection frequency of different schemes based on average of 1000 iterations per packet delay threshold. We have included a $20 \%$ hysteresis for static, Markov chain and moving average schemes for additional stability of their selection outcomes. From the results in Fig. 8, we remark that our algorithm is superior in precluding 'ping pong' effect with the lowest network re-selection frequency even without additional hysteresis control. In particular, our algorithm yields a significant improvement of at least twofold when the packet delay threshold is near the gray decision zone vunerable to other selection schemes.

\section{CONCLUSion ANd Future Work}

We have presented an novel dynamic network selection algorithm that could be used to estimate dynamic QoS information such as packet delay, packet loss and jitter, thereby exploiting them to prevent unnecessary handovers with sequential Bayesian estimation. These QoS information are critical for real-time multimedia traffic which would be predominant in $4 \mathrm{G}$ networks. As proof-of-concept, we have demonstrated the practicality of our dynamic network selection algorithm which is efficient in capturing dynamic QoS information and effective in providing stable network selection. The deployment of DAPU in BS/AP would be seamless since it require no architectural changes to existing infrastructures and implementation of sequential Bayesian estimation in MT is highly feasible as a virtue of its low computational complexity.

For future work, we planned to extend our algorithm to consider multiple QoS parameters and access networks. The consideration of qualitative information from user preference is also under development to produce a truly $\mathrm{ABC}$ decision together with the quantitative information collected via our DAPU. QoS performance metrics before and after handover based on ABC decision will be also investigated. Finally, a decentralized load balancing function will be incorporated with our DAPU to ensure that handovers would result in optimal traffic distributions in pervasive networks.

\section{REFERENCES}

[1] E. Gustafsson and A. Jonsson. Always best connected. IEEE [see also IEEE Personal Communications] Wireless Communications, 10(1):4955, February 2003.

[2] J. M. Pereira. Fourth generation: now, it is personal! In Personal, Indoor and Mobile Radio Communications, 2000. PIMRC 2000. The 11th IEEE International Symposium on, volume 2, pages 1009-1016, London, September 2000.

[3] Chen Yiping and Yang Yuhang. A new 4g architecture providing multimode terminals always best connected services. IEEE [see also IEEE Personal Communications] Wireless Communications, 14(2):3641, April 2007.

[4] Quoc-Thinh Nguyen-Vuong, Nazim Agoulmine, and Yacine GhamriDoudane. Terminal-controlled mobility management in heterogeneous wireless networks. IEEE Communications Magazine, 45(4):122-129, April 2007.

[5] Qingyang Song and Abbas Jamalipour. An adaptive quality-of-service network selection mechanism for heterogeneous mobile networks: Research articles. Wirel. Commun. Mob. Comput., 5(6):697-708, 2005.

[6] Qian Zhang, Chuanxiong Guo, Zihua Guo, and Wenwu Zhu. Efficient mobility management for vertical handoff between WWAN and WLAN IEEE Communications Magazine, 41(11):102-108, November 2003.

[7] John F. Gibbon. Real-time scheduling for multimedia services using network delay estimation. PhD thesis, Boston University, Boston, MA, USA, 1994.

[8] Bradley Efron and Robert J. Tibshirani. An Introduction to the Bootstrap. Chapman \& Hall, 1993.

[9] A. M. Zoubir and B. Boashash. The bootstrap and its application in signal processing. IEEE Signal Processing Magazine, 15(1):56-76, January 1998

[10] Andrew Gelman, John B. Carlin, Hal S. Stern, and Donald B. Rubin. Bayesian Data Analysis, Second Edition. Chapman \& Hall, 2003.

[11] G. Bianchi and I. Tinnirello. Kalman filter estimation of the number of competing terminals in an IEEE 802.11 network. In INFOCOM 2003. Twenty-Second Annual Joint Conference of the IEEE Computer and Communications Societies. IEEE, volume 2, pages 844-852, March/April 2003.

[12] J. G. Atallah and M. Ismail. Future 4g front-ends enabling smooth vertical handovers. IEEE Circuits and Devices Magazine, 22(1):6-15, January/February 2006.

[13] F. Anjum, M. Elaoud, D. Famolari, A. Ghosh, R. Vaidyanathan, A. Dutta, P. Agrawal, T. Kodama, and Y. Katsube. Voice performance in WLAN networks - an experimental study. In Global Telecommunications Conference, 2003. GLOBECOM '03. IEEE, volume 6, pages 3504-3508, December 2003.

[14] H. Cramer. Mathematical Methods of Statistics. Princeton University Press, 1946.

[15] Chie Ming Chou and ChingYao Huang. Dynamic vertical handover control algorithm for WLAN and UMTS. 2006. WCNC 2006. IEEE Wireless Communications and Networking Conference, 1:606-610, April 2006. 